\title{
A Pedagogical Borderland? Comparing Student and Faculty Attitudes and Actions About Teaching and Learning
}

\section{Dr. Edward J. Berger, Purdue University, West Lafayette}

Edward Berger is an Associate Professor of Engineering Education and Mechanical Engineering at Purdue University, joining Purdue in August 2014. He has been teaching mechanics for nearly 20 years, and has worked extensively on the integration and assessment of specific technology interventions in mechanics classes. He was one of the co-leaders in 2013-2014 of the ASEE Virtual Community of Practice (VCP) for mechanics educators across the country.

\section{Mr. Ryan R. Senkpeil, Purdue Engineering Education}

Ph.D. student in Engineering Education at Purdue University. My research interests include: non-cognitive factors and their impact on student academic performance, non-cognitive factors and engineering student retention, and personalized intervention design.

I have previous research experience in engineering education working with first year engineering teams and analyzing peer evaluation comments. I also have experience in solar physics research through the Harvard Smithsonian Center for Astrophysics.

\section{Elizabeth K. Briody Ph.D., Cultural Keys LLC}

I am a cultural anthropologist whose areas of expertise include work culture and organizational-culture change. I founded Cultural Keys LLC in 2009, a consulting firm specializing in improving organizational effectiveness, and understanding and reaching customers. I have consulted in a wide variety of industries including health care, apparel, insurance, and automotive. Prior to that, I worked for many years at General Motors R\&D, first as a Senior Research Scientist and ultimately as a Technical Fellow. Two of my recent books are The Cultural Dimension of Global Business (with G. Ferraro - 2013, 7th ed., Pearson) and Transforming Culture (with R.T. Trotter, II and T.L. Meerwarth - 2014, Palgrave).

\section{Edward F. Morrison, Purdue University, West Lafayette}

Ed Morrison is Regional Economic Development Advisor for the Center for Regional Development at Purdue University. Ed has been developing a new approach to developing strategies for complex collaboration in open, loosely connected networks. Called "strategic doing", this methodology emphasizes the strategic value of collaboration in today's global economy. For over twenty-five years, he conducted strategy projects throughout the U.S. His work won the first Arthur D. Little Award for excellence in economic development presented by the American Economic Development Council. Prior to starting his economic development work, Ed worked for Telesis, a corporate strategy consulting firm. In this position, he served on consulting teams for clients such as Ford Motor Company, Volvo, and General Electric. He conducted manufacturing cost studies in the U.S., Japan, Mexico, Canada, Italy, Sweden, and France. Ed started his professional career in Washington, D.C., where he has served as a legislative assistant to an Ohio Congressman, staff attorney in the Federal Trade Commission, and staff counsel in the US Senate. He holds a BA degree cum laude with honors from Yale University and MBA and JD degrees from the University of Virginia. 
WIP: A Pedagogical Borderland? Comparing Student and Faculty Attitudes and Actions About Teaching and Learning

\section{Introduction}

This Work in Progress (WIP) paper describes early results from a new research study on differences in attitudes and actions between students and instructors, and their impact on academic outcomes. In anthropology, a 'borderland' is a physical or metaphorical region in which two cultures, ideas, or sets of values meet and interact, with a new borderland culture (related to, but distinct from, either of the two original cultures) emerging as a result. This research seeks to characterize the pedagogical borderland of a higher education engineering classroom, into which students and faculty bring a set of attitudes, beliefs, history and so forth that drive their behaviors and choices in the learning environment - and of course influence student academic outcomes as well. For students who consented to participate in this study $\left(n_{s}=317\right)$, we delivered the 44-item FelderSoloman Index of Learning Styles (ILS) ${ }^{[1]}$, the 10 -item Big Five personality inventory ${ }^{[2]}$, the 8-item grit survey ${ }^{[3]}$, the Motivated Strategies for Learning Questionnaire (MSLQ) 13-item subscale on study skills and test anxiety ${ }^{[4]}$, and we also obtained their academic transcript and admissions data. For faculty who consented $\left(n_{f}=33\right)$, we delivered the 44item ILS, the 16-item Approaches to Teaching Inventory (ATI) ${ }^{[5]}$, and a 19-item Pedagogical Inventory (PI) that asked faculty to indicate their level of awareness and use of specific pedagogical tools (active learning approaches, lecturing, think-pair-share, etc.) in their teaching. For a particular sophomore-level course, we matched ILS scores of students with their instructor in that course, and calculated an ILS misalignment score between the students and their instructor across all four ILS sub-scales. Our hypothesis was that a misalignment between faculty and student ILS scores would result in worse academic outcomes for students (as measured by course final letter grade), even when controlling for prior performance in an important prerequisite course. Our preliminary results suggest this hypothesis to be true, although only weakly so. The entire picture of student performance is much more complicated, and this work in progress paper is just the beginning of a long-term research trajectory that seeks to understand pedagogical borderlands and how students and faculty navigate them.

\section{Motivation}

We are interested in understanding the alignment between student and faculty ideas, attitudes, beliefs, and preferences about teaching and learning. Our research question to begin this investigation is: to what extent does misalignment in ideas, beliefs, or attitudes between faculty and students explain student academic outcomes? Our hypothesis is that more misalignment between faculty and students results in worse student outcomes, on average. This is our first set of data analyses to pursue this question, and before deploying the survey we hypothesized several potential ways to characterize 'misalignment'. This is a long-term research trajectory, and the results reported here will be substantially extended in the coming years as we collect more data, explore alternative definitions of misalignment, and use more sophisticated techniques to analyze the data. 


\section{Participants}

We collected survey responses from faculty $\left(n_{f}=33\right)$ and students $\left(n_{s}=317\right)$ affiliated with a mechanical engineering program at a large Midwestern research-intensive university. We then chose a particular sophomore level required course (dynamics) as our test bed for beginning exploration of the research question. Of the 33 faculty respondents, 4 had taught dynamics in the recent past. Of the 317 student respondents, 110 had already completed dynamics with one of the four faculty who had recently taught dynamics (and for whom we have survey responses). As a result, we can match student survey responses with their instructor's responses, and compare those survey responses in light of student grades in the course. Each ILS dimension is scored on a $(-11,11)$ interval describing the spectrum between two extremes. For example, a score of $(-11)$ on the ACT-REF dimension indicates a strong preference for active learning, while a score of $(+11)$ suggests a deeply reflective learner. In this first phase of data analysis, we define misalignment as the (student) - (instructor) ILS score across all four ILS dimensions. As such, we have 440 misalignment scores (4 ILS dimensions for each of 110 students), and all are integers on the interval $(-22,22)^{[1]}$. We also have dynamics course grade for each student.

\section{Results-ILS Misalignment}

When we consider all participants from both the faculty $\left(n_{f}=33\right)$ and student $\left(n_{s}=317\right)$ populations, there is a misalignment between their ILS sub-scales, with two of them being different in statistically significant ways (Table 1). In particular, students are more sensing learners as compared with faculty (SEN-INT sub-scale: $p<0.0001)$, and they are more active learners (ACT-REF sub-scale: $p<0.001$ ). On the other two ILS sub-scales, faculty and students are not statistically significantly different. These differences may reflect known differences between novices and experts. Novices (students) may be at the stage of intellectual development in which they prefer procedural knowledge (SEN), while experts (faculty) have cultivated their conceptual knowledge (INT) to shape meaning. Similarly, novices may prefer an exploratory type of learning (ACT), while experts have the experience and knowledge to be more reflective and engage in deep thinking (REF) about problems of significance.

Table 1. ILS comparison for students $\left(n_{s}=317\right)$ and faculty $\left(n_{f}=33\right)$.

\begin{tabular}{llll}
\hline ILS Dimension & $\begin{array}{l}\text { Student score, } \\
\text { mean }(\mathrm{sd})\end{array}$ & $\begin{array}{l}\text { Faculty score, } \\
\text { mean }(\mathrm{sd})\end{array}$ & $\begin{array}{l}\text { p-value for mean } \\
\text { difference }\end{array}$ \\
\hline ACT-REF & $-0.92(4.73)$ & $2.30(4.24)$ & $<0.001$ \\
SEN-INT & $-1.89(5.07)$ & $2.12(5.08)$ & $<0.0001$ \\
VIS-VER & $-6.15(3.90)$ & $-5.27(5.15)$ & 0.236 \\
SEQ-GLO & $-1.31(4.14)$ & $0.12(5.02)$ & 0.065 \\
\hline
\end{tabular}

The implications of this misalignment have been explored using data from each of the 110 students in dynamics and their 4 instructors. A misalignment score for each student was calculated by comparing their ILS profile to their instructor's. For each ILS dimension, the average grade for students at each value of misalignment on that ILS dimension was calculated, and the aggregate results are presented in Figure 1. The four sub-figures correspond to the four ILS dimensions, and a regression line has been plotted 
on each sub-figure. Of the four ILS dimensions, only the SEQ-GLO dimension regression has any significance $[F(1,12)=10.71, p=0.0067)$, and in fact this result suggests that not only does student-faculty alignment on this ILS dimension matter in determining a student's course grade, but the direction (i.e., sign) of the (mis-)alignment matters. The sub-figure shows that students who are more sequentially oriented than their instructor generally earned better grades than their peers who are highly aligned (misalignment score of 0 ) or who are more globally oriented than their instructor.

We explored whether this result could be explained by student ILS score alone along the SEQ-GLO dimension, with the idea that perhaps more sequentially-oriented learners do better in the course in general. This turned out not to be the case, and the resulting plot (not shown) of grade against student ILS (SEQ-GLO) score was essentially a cloud of points through which no statistically significant regression line could be drawn. So it appears that student-instructor mismatch along the SEQ-GLO dimension is influential in student grade, and we continue to explore the reasons behind this in more depth.

\section{Discussion-A Pedagogical Borderland?}

There are three interesting observations that we can make so far. First, there is indeed a misalignment between faculty and students in the aggregate within the populations sampled here, and along two of those ILS dimensions the misalignment is statistically significant (Table 1). Second, this misalignment apparently does have a relationship to course performance, at least along the SEQ-GLO dimension of the ILS (Figure 1). Third, this data (despite the statistically significant relationship along the SEQ-GLO dimension) strongly suggests that students can achieve good performance in the dynamics class we studied, regardless of misalignment (Figure 1). The conclusion here is that there is indeed a borderland defined by ILS mismatch in the classroom, and that students' abilities to navigate that borderland can result in strong academic performance. However, ILS mismatch alone does not present a full picture of the borderland, and other dimensions of misalignment must be explored to expand our understanding.

But we also acknowledge an obvious criticism of the work so far: we are measuring misalignment along a learning-learning axis for students and instructors. That is, we are comparing student learning styles with faculty learning styles. Embedded in this initial work is the hypothesis that faculty learning styles influence their teaching attitudes and behaviors. While this notion has some intuitive appeal, it is far from conclusively proven, and in fact another obvious criticism is the question of what constructs the ILS actually probes. We understand that the ILS is viewed as imperfect, but we have nonetheless used the instrument largely as a mechanism of comparison (not as an absolute measure of learning preferences) between students and instructors as a starting point for this research. With all that said, it seems clear that characterizing misalignment along a learning-teaching axis would likely add great value to the analysis we have done already and further explain the borderland phenomenon. The data we collected from faculty on the Approaches to Teaching Inventory and the Pedagogical Index enable analysis on a learning-teaching axis, and this and several other on-going research efforts are described next. 


\section{Emergent research questions and on-going activity}

This work is the first stage of analysis of the data. The next set of research questions we are preparing to tackle includes a closer look at the misalignment question, specifically:

- RQ1. To what extent does misalignment along other learning-teaching axes further explain student academic performance? Working hypothesis: Variations in instructor beliefs (as measured by the ATI) or in-class actions (as measured by the PI) will further explain student academic performance because misalignment along the learning-teaching axis is more influential than misalignment along the learning-learning axis.

- $\quad$ RQ2. In what ways can the learning-teaching alignment be characterized? Working hypothesis: Mapping between the ILS and the ATI/PI results is not immediately obvious, and we are currently exploring a variety of metrics to capture learning-teaching misalignment in a quantitative way.

- RQ3. To what extent does learning-teaching (or learning-learning) misalignment explain faculty teaching evaluations? Working hypothesis: in the same way that misalignment creates consequences for student academic performance, it also has a measureable effect on faculty teaching evaluations. That is, we believe that students perceive the misalignment and evaluate their instructors accordingly.

\section{Acknowledgement}

This material is based upon work supported by the National Science Foundation under Grant No. EEC-1519412 and the Revolutionizing Engineering Departments (RED) program. Any opinions, findings, and conclusions or recommendations expressed in this material are those of the authors and do not necessarily reflect the views of the National Science Foundation.

\section{References}

[1] T. A. Litzinger, S. H. Lee, J. C. Wise, and R. M. Felder, "A Psychometric Study of the Index of Learning Styles,” J. Eng. Educ., vol. 96, no. 4, pp. 309-319, 2007.

[2] S. D. Gosling, P. J. Rentfrow, and W. B. Swann, "A very brief measure of the Big-Five personality domains," J. Res. Pers., vol. 37, no. 6, pp. 504-528, Dec. 2003.

[3] A. L. Duckworth and P. D. Quinn, "Development and Validation of the Short Grit Scale (Grit-S)," J. Pers. Assess., vol. 91, no. 2, pp. 166-174, 2009.

[4] P. R. Pintrich, D. A. F. Smith, T. Garcia, and W. J. McKeachie, "Reliability and predictive validity of the Motivated Strategies for Learning Questinnaire (MSLQ)," Educ. Psychol. Meas., vol. 53, pp. 928-940, 1999.

[5] K. Trigwell and M. Prosser, "Development and Use of the Approaches to Teaching Inventory," Educ. Psychol. Rev., vol. 16, no. 4, pp. 409-424, 2004. 

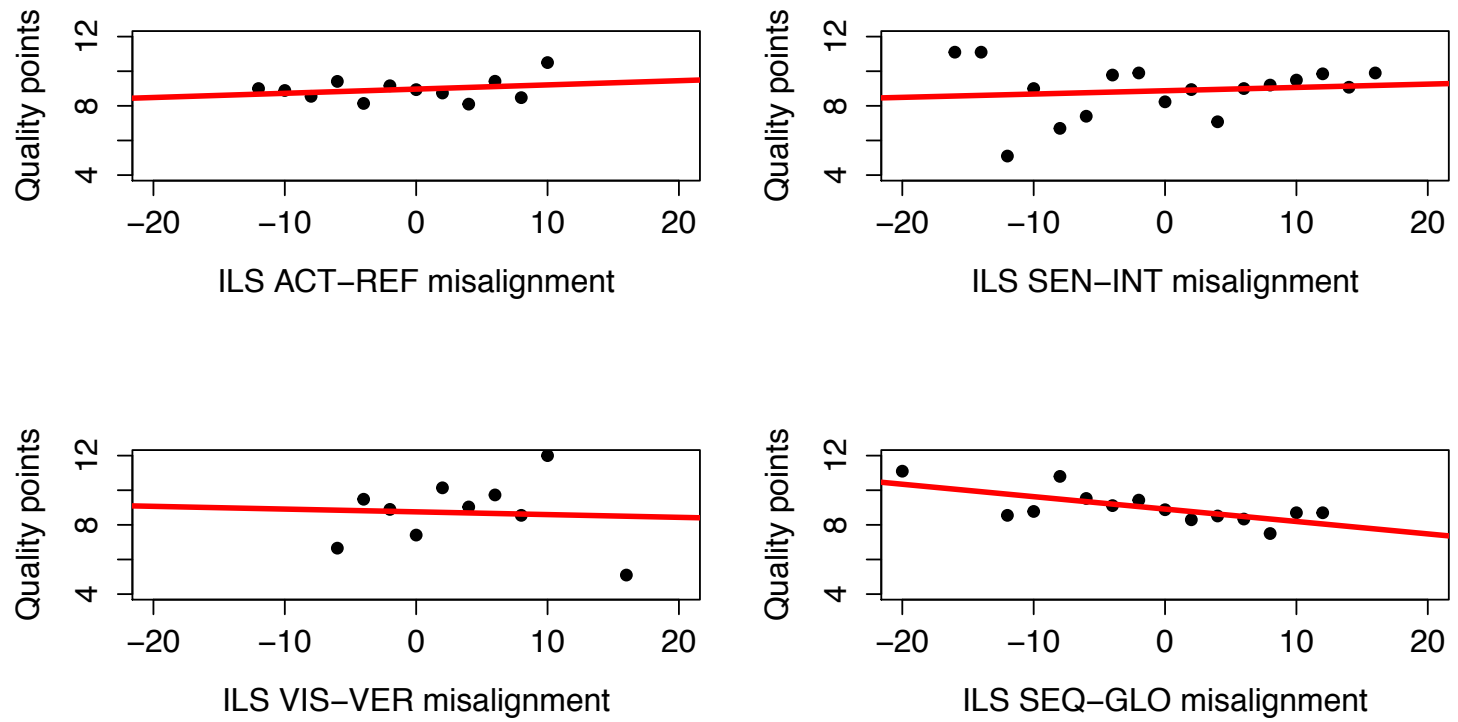

Figure 1. Dynamics grade as a function of student-instructor misalignment along the 4 ILS dimensions. Data is plotted as 'quality points' (QP), where 12 QP corresponds to a grade of A, 9 corresponds to a $\mathrm{B}$, and so forth. 